\title{
Bacterial Resistance Pattern to Cephalosporin in Patient with Chronic Osteomyelitis at Dr. Hasan Sadikin General Hospital Bandung
}

\author{
Daniel Christian Fernandez, ${ }^{1}$ Ghuna Arioharjo Utoyo, ${ }^{2}$ Adhi Kristianto Suganli ${ }^{3}$ \\ ${ }^{1}$ Faculty of Medicine Universitas Padjadjaran, ${ }^{2}$ Department of Orthopaedic and Traumatology \\ Faculty of Medicine Universitas Padjadjaran/Dr. Hasan Sadikin General Hospital Bandung, \\ ${ }^{3}$ Department of Clinical Pathology Faculty of Medicine Universitas Padjadjaran/Dr. Hasan Sadikin \\ General Hospital Bandung
}

\begin{abstract}
Background: Chronic osteomyelitis remains difficult to treat and has a high rate of relapse after apparently succesful treatment. Cephalosporin has been greatly used for the antibiotic intervention for patient with osteomyelitis. The objective of this study was to explore bacterial profile and its microbial resistance pattern of each generation of cephalosporin from patients with chronic osteomyelitis at Dr. Hasan Sadikin General Hospital.

Methods: A descriptive study was conducted to 39 medical records from patients with chronic osteomyelitis at Dr. Hasan Sadikin General Hospital that met both inclusion and exclusion criteria. The bacterial pattern and antimicrobial susceptibility test results were obtained from both medical record and laboratory database for five years, during the period of Januari 2009 to December 2014. Data of bacterial species, type of Gram staining, and numbers of resistance isolates to antiobiotic were taken from the collected data and analyzed in form of frequency tabulation and percentages.

Results: As high as 43-52\% of Staphylococcus aureus isolates were resistant to all generation of cephalosporin. Coagulase-negative Staphylococci and Streptococcus spp. showed resistance to cephalosporin as high as 29\% and 67\%, respectively. This study also discovered that 5 of 23 Staphylococcus aureus isolates were methicillin-resistant Staphylococcus aureus (MRSA). Klebsiella pneumoniae showed a leading number of resistance pattern for both third and fourth generation of cephalosporin (77\%). Enterobacter cloacae showed highly resistance for both first and third generation of cephalosporin, between 57-86\%.

Conclusions: There is the high resistance of all bacteria to cephalosporin among chronic osteomyelitis patients. [AMJ.2016;3(3):376-81]
\end{abstract}

Keywords: Antimicrobial resistance, cephalosporin, chronic osteomyelitis

\section{Introduction}

Chronic osteomyelitis is one of the most challenging and expensive diseases to treat, despite advances in antibiotics and new operative intervention techniques. ${ }^{1}$ Specific diagnostic strategies are ill-defined and lack of prospective evidence. It is difficult to detect the acute phase symptoms of osteomyelitis, therefore, it is more likely to treat the patient when the disease progresses to the chronic phase. ${ }^{2}$ The gold standard to establish a diagnosis remains by a bone culture, but falsenegative rates are reported as high as $40 \%{ }^{3}$ The invasive procedure has to be conducted to take the sample for microbial culture, and it is more likely having a delay since it is commonly taken during surgical procedure e.g adjacent tissue reconstruction, drainage, and repair. As the result, the patient is given an empiric antibiotic treatment before knowing the etiological agent for the disease. Studies showed that patient receiving antibiotic regimen without bone culture-guidance is more likely to have worst outcome than those with any bone culture-guided antibiotic regimen. ${ }^{4}$

In the antibiotic era, chronic osteomyelitis remains difficult to treat and has a high rate of relapse after apparently succesful treatment. ${ }^{1,3}$ Since it has the same characteristic as penicillin for antimicrobial treatment in osteomyelitis, cephalosporin has been greatly used for the antibiotic intervention to kill bacteria that infect the patient with osteomyelitis. 5,6 Klebsiella pneumoniae, as one of the etiological agents of chronic osteomyelitis, was reported to be resistant as high as $44 \%$ to third generation of cephalosporin. ${ }^{7}$ In the

Correspondence: Daniel Christian Fernandez, Faculty of Medicine, Universitas Padjadjaran, Jalan Raya Bandung-Sumedang Km.21, Jatinangor, Sumedang, Indonesia, Phone: +6285276077912 Email: danielchristianfernandez@gmail.com 
United States, patients with the infection due to antibacterial-resistance organism, has a higher cost of treatment compared to patients with antibacterial-susceptible organism. ${ }^{8}$ The increase of bacterial resistance gives a great influence on mortality rate, substantial cost of the treatment, and the length of stay in hospital..$^{9,10}$

The objective of this study was to explore bacterial profile and its microbial resistance pattern of each generation of cephalosporin from patients with chronic osteomyelitis at Dr. Hasan Sadikin General Hospital, Bandung, Indonesia.

\section{Methods}

The descriptive design was chosen to accomplish this study that was performed at Dr. Hasan Sadikin General Hospital. The population of the study was taken from all patients' medical records which had been diagnosed as having osteomyelitis and admitted to hospital for five years during the period of Januari 2009 to December 2014.

The inclusion criterion of this study was all patient medical records admitted to Dr. Hasan Sadikin General Hospital for the first time and diagnosed to have chronic ostemyelitis, either as primary diagnosis or additional diagnosis. The exclusion criteria of this study were chronic osteomyelitis patients without complete nor available antimicrobial susceptibility testing result. The data collection process was divided into two procedures.The first procedure was gathering all the primary information about patient's demographic profile with chronic osteomyelitis. The first procedure was taken from digital medical record database with disease group categories: chronic osteomyelitis (diagnosis code: M86.6), according to International Classification of Diseases (ICD) 10. After getting the lists of patient and medical record number, full set hard copies of each patient's medical record was taken and evaluated for inclusion criteria. The second procedure was matching the first admission of patient with the date of the sample sent to laboratory for antimicrobial susceptibility testing from laboratory database system. From Januari 2009 to December 2014, there were 332 patients with chronic osteomyelitis who had been admitted to Dr. Hasan Sadikin General Hospital. Among them, there were only 98 patients with chronic osteomyelitis, which had complete medical record. From 98 patients with complete medical record, there were only 39 patients who had antimicrobial

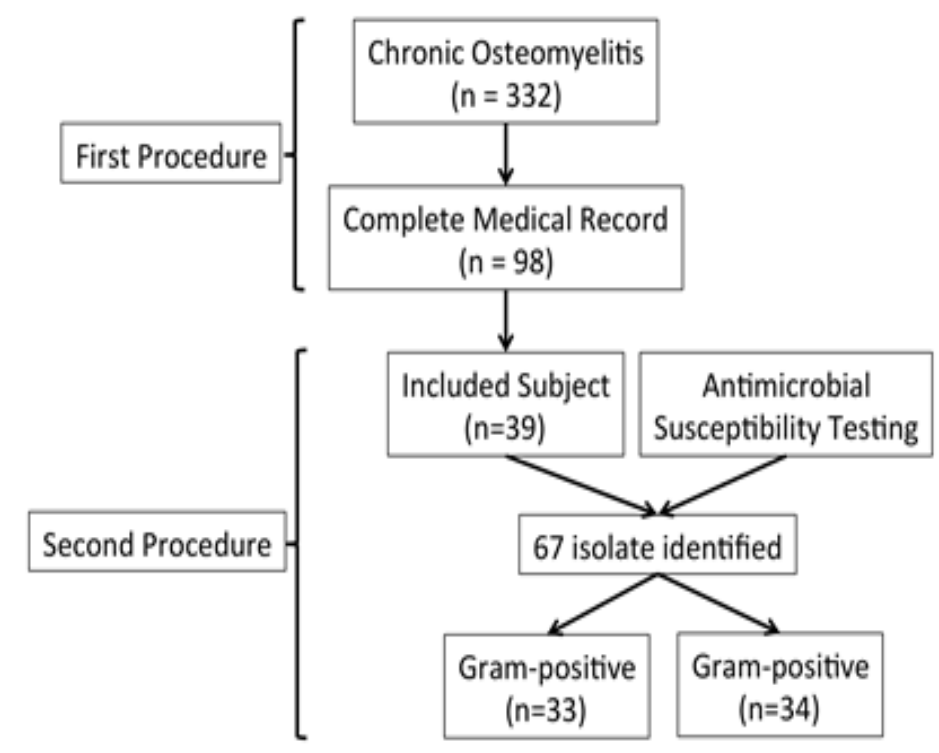

Figure 1 The Study Procedure Chart

Althea Medical Journal. 2016;3(3) 
susceptibility testing result, in concordance with first admission of patient. There were 67 bacteria identified from the 39 patients with chronic osteomyelitis (Figure 1).

Data of bacterial species, types of Gram staining, numbers of resistance isolates to antiobiotic were taken and analyzed in form of frequency tabulation and percentages. This study was conducted with the ethical standard approval from both Faculty of Medicine Universitas Padjadjaran and Dr. Hasan Sadikin General Hospital, with ethical letter No. LB.04.01/A05/EC/312/VII/2015.

\section{Results}

The distribution of bacteria isolated from chronic osteomyelitis was described in Table 1. In this study, the most Gram-positive bacteria isolated were Staphylococcus aureus and the most Gram-negative bacteria isolated were Klebsiella pneumoniae.

Table 2 shows the number of bacteria as the etiology of chronic osteomyelitis with the antimicrobial susceptibility testing. Based on the result, almost all bacteria were resistant to cephalosporin group. Cephazoline, as a part of first generation of cephalosporin, had a high range of resistance pattern for both Gram-positive and Gram-negative bacteria, with a value between $29-86 \%$. As high as $86 \%$ of Enterobacter cloacae were resistant to cephazoline and had the highest resistance pattern compared to any bacteria to the first generation of cephalosporin. Cefadroxil, which was tested only to Grampositive bacteria, had a range of resistance between 13-33\%. Meanwhile, the second generation of cephalosporintested only for the Gram-positive bacteria, showed both cefoxitin and cefuroximehave resistance value of aprroximately $48 \%$ and between $29-48 \%$ respectively.

In third generation of cephalosporin, there were ceftriaxone, ceftazidime, and cefotaxime that were tested for both Gram-positive and Gram-negative bacteria. Ceftriaxone had a high pattern of resistance, with a value range between 29-77\%. Ceftazidime had the same high resistance pattern as ceftriaxone, with a value range between 29-77\%. Meanwhile, there were only slight differences of resistance pattern between ceftriaxone and ceftazidime found in Gram-negative bacteria.

Even though cefotaxime had an absence of resistance pattern for the major etiological bacteria, italso had the highest resistance pattern for the minor etiological bacteria, both Gram-positive and Gram-negative, with a value range between 33-67\%. Cefoperazone and cefotaxime, as parts of third generation of cephalosporin tested only for Gram-positive bacteria, had high class of resistant pattern, with value range between $29-67 \%$ and $33-35 \%$, respectively. In fourth generation of cephalosporin, cefepime used to test the resistance pattern of bacteria for both positive and negative bacteria, showed a high resistance pattern, with a value range between $29-77 \%$.

Table 1 Bacteria Isolated from 39 Patients with Chronic Osteomyelitis

\begin{tabular}{lcc}
\hline \multicolumn{1}{c}{ Bacteria } & $\begin{array}{c}\text { Numbers of isolates } \\
(\mathbf{n = 6 7 )}\end{array}$ & Percentages (\%) \\
\hline Gram-positive & - & 34.3 \\
Staphylococcus aureus & 23 & 10.4 \\
Coagulase-negative Staphyloccocus & 7 & 4.4 \\
Streptococcus spp. & 3 & - \\
Gram-negative & - & 10,4 \\
Enterobacter cloacae & 7 & 7.4 \\
Escherichia coli & 5 & 13.4 \\
Klebsiella pneumoniae & 9 & 2.9 \\
Morganella morganii & 2 & 4.4 \\
Proteus mirabilis & 3 & 8.9 \\
Pseudomonas aeruginosa & 6 & 2.9 \\
Serratia marcescens & 2 & -4 \\
\hline
\end{tabular}




\begin{tabular}{|c|c|c|c|c|c|c|c|c|c|c|}
\hline Antibiotics & 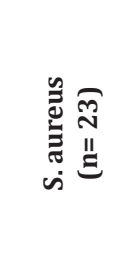 & 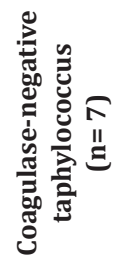 & 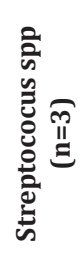 & 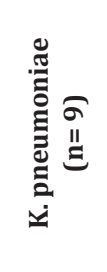 & 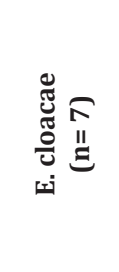 & 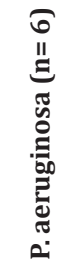 & 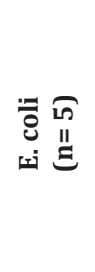 & 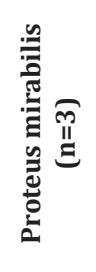 & 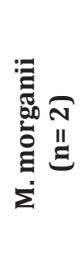 & 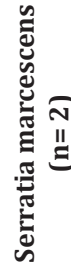 \\
\hline & $\% \mathrm{R}$ & $\% \mathrm{R}$ & $\% \mathrm{R}$ & $\% \mathrm{R}$ & $\% \mathrm{R}$ & $\% \mathrm{R}$ & $\% \mathrm{R}$ & $\% \mathrm{R}$ & $\% \mathrm{R}$ & $\% \mathrm{R}$ \\
\hline \multicolumn{11}{|l|}{$\beta$-lactam/ with adjuvant } \\
\hline Amoxicillin-Clav & $1(4.3)$ & - & - & $4(44.4)$ & - & $3 / 6$ & - & $1 / 3$ & $2 / 2$ & - \\
\hline Ampicillin-Sulbactam & - & - & - & $3(33.3)$ & $4(57.1)$ & $5 / 6$ & $5 / 5$ & $1 / 3$ & $2 / 2$ & $2 / 2$ \\
\hline Oxacillin & $6(26.0)$ & - & - & - & - & - & - & - & - & - \\
\hline Piperacillin-Tazobactam & $3(13.0)$ & - & - & $5(55.6)$ & $2(28.5)$ & - & - & $1 / 3$ & - & - \\
\hline \multicolumn{11}{|l|}{ Cephalosporin } \\
\hline Cefadroxil* & $3(13.0)$ & - & $1 / 3$ & - & - & - & - & - & - & - \\
\hline Cefazoline* & $10(43.4)$ & $2(28.5)$ & $2 / 3$ & $4(44.4)$ & $6(85.7)$ & $3 / 6$ & $1 / 5$ & $1 / 3$ & $1 / 2$ & $2 / 2$ \\
\hline Cefoxitine** & $11(47.8)$ & - & - & - & - & - & - & - & - & - \\
\hline Cefuroxime** & $11(47.8)$ & $2(28.5)$ & $1 / 3$ & - & - & - & - & - & - & - \\
\hline Cefoperazone ${ }^{* * *}$ & $11(47.8)$ & $2(28.5)$ & $2 / 3$ & - & - & - & - & - & - & - \\
\hline Ceftriaxone ${ }^{* * *}$ & $12(52.1)$ & $2(28.5)$ & $2 / 3$ & $7(77.8)$ & $5(71.4)$ & $2 / 6$ & $2 / 5$ & $2 / 3$ & - & - \\
\hline Ceftazidime*** & $12(52.1)$ & $2(28.5)$ & $2 / 3$ & $7(77.8)$ & $4(57.1)$ & $3 / 6$ & $2 / 5$ & $1 / 3$ & - & $1 / 2$ \\
\hline Cefixime*** & $8(34.7)$ & - & $1 / 3$ & - & - & - & - & - & - & - \\
\hline Cefotaxime*** & - & - & $2 / 3$ & - & - & $2 / 6$ & - & $1 / 3$ & - & - \\
\hline Cefepime ${ }^{* * * *}$ & $11(47.8)$ & $2(28.5)$ & $2 / 3$ & $7(77.8)$ & $2(28.5)$ & $2 / 6$ & $1 / 5$ & $2 / 3$ & - & - \\
\hline \multicolumn{11}{|l|}{ Carbapenem } \\
\hline Imipenem & $11(47.8)$ & $2(285)$ & - & - & - & $1 / 6$ & - & - & - & - \\
\hline Meropenem & $12(52.1)$ & $2(28.5)$ & - & $1(11.1)$ & - & $1 / 6$ & - & - & - & - \\
\hline \multicolumn{11}{|l|}{ Aminoglycoside } \\
\hline Amikacin & - & - & - & $2(22.2)$ & - & - & - & - & - & - \\
\hline Gentamycin & $10(43.4)$ & $2(28.5)$ & - & $6(66.7)$ & $3(42.8)$ & $4 / 6$ & $1 / 5$ & $2 / / 3$ & $1 / 2$ & - \\
\hline \multicolumn{11}{|l|}{ Tetracyclins } \\
\hline Tetracyclin & $3(13.0)$ & - & - & - & - & - & - & $2 / 3$ & - & - \\
\hline Tigecyclin & - & - & - & $2(22.2)$ & - & $6 / 6$ & - & $3 / 3$ & $2 / 2$ & - \\
\hline \multicolumn{11}{|l|}{ Macrolides } \\
\hline Erythromycin & $10(43.4)$ & $1(14.2)$ & $1 / 3$ & - & - & - & - & - & - & - \\
\hline \multicolumn{11}{|l|}{ Oxazolidonones } \\
\hline Linezolid & $1(4.34)$ & - & - & - & - & - & - & - & - & - \\
\hline \multicolumn{11}{|l|}{ Fluoroquinolone } \\
\hline Ciprofloxacin & $10(43.4)$ & $3(42.8)$ & $2 / 3$ & $6(66.7)$ & $3(42.8)$ & $3 / 6$ & $4 / 5$ & $2 / 3$ & $2 / 2$ & - \\
\hline Levofloxacin & $10(43.4)$ & $2(28.5)$ & - & $5(55.6)$ & $3(42.8)$ & $3 / 6$ & $3 / 5$ & $1 / 3$ & $1 / 2$ & - \\
\hline Moxifloxacin & $3(13.0)$ & $1(14.2)$ & - & - & - & - & - & - & - & - \\
\hline \multicolumn{11}{|l|}{ Folate Pathway Inhibitors } \\
\hline Cotrimoxazole & $7(30.4)$ & $1(14.2)$ & $2 / 3$ & $6(66.7)$ & $3(42.8)$ & $2 / 6$ & $2 / 5$ & $2 / 3$ & - & - \\
\hline
\end{tabular}




\section{Discussions}

Chronic osteomyelitis, though uncommon, causes serious morbidity such as permanent disability, paralysis, and rarely death. Management of this disease can be challenging for many physicians, mainly due to an unfamiliarity and poor evidence base. ${ }^{11}$ Despite the treatment, diagnosing chronic osteomyelitis faces a dilemma of the accepted range of time to give an empirical antibiotic treatment before conducting the invasive procedure to take sample for culture test. The exposure of culture-nonguided antibiotic seems to increase the resistance pattern of bacteria. More recent study showed that the increased bacterial resistance result suggested the changing of the microbiology of this disease. ${ }^{12}$

This study discovered that infection caused by Gram-positive bacteria was relatively proportional compared to Gram-negative bacteria (49\% versus 51\%). Staphylococcus aureus was found in 23 isolates or over one third of microbial culture. As comparison, recent studies showed that Staphylococcus species was the most common pathogen responsible for osteomyelitis.12-14 Staphylococcus aureus is the typical pathogen responsible for both acute and chronic ostemyelitis, and it has great potential to develop antimicrobial resistance and virulence factor expression, regardless of patient's immune status. Due to its high virulence, Staphylococcus aureus may cause several complications, from localized superficial infection to the most severe forms of bacteremia, such as septic arthritis, endocarditis, and septic shock syndrome. This situation becomes more complex due to high incidence of methicillinresistant Staphylococcus aureus (MRSA). ${ }^{14}$ This study also discovered that 5 of 23 isolates Staphylococcus aureus were methicillinresistant Staphylococcus aureus (MRSA). Methicillin-resistant Staphylococcus aureus (MRSA) appears to be biologically succesful and commonly causes severe soft tissue infections, complicated by musculoskeletal involvement in some cases. ${ }^{11}$

Almost half of Staphylococcus aureus isolates were resistant to all generation of cephalosporin, between $43-52 \%$, followed by coagulase-negative Staphylococci and Streptococcus spp. with $29 \%$ and $67 \%$ of overall percentage resistance, respectively. It also showed that Staphylococcus aureus was more susceptible to penicillin with adjuvant. As predicted from a recent study by
Tuchscherr et al. ${ }^{15}$, it showed that the majority of beta-lactam group lose activities against Staphylococcus aureus.

Klebsiella pneumoniae and Enterobacter cloacae were the first and second most common Gram-negative bacteria found in cultures with $13.4 \%$ and $10.4 \%$, respectively. Gramnegative bacteria have grown in importance as causative agents due to the increasing number of orthopaedic surgeries related to fractures with the use of implants. ${ }^{16}$ As used in recent study by Kevin et al. ${ }^{17}, 10 \%$ of resistance pattern were classified as the low resistance pattern, and $10 \%$ or more of resistance pattern were termed as high resistance pattern. This resistance classification was used to identify the degree of the resistance pattern.

Based on the study, Klebsiella pneumoniae showed the leading number of resistance pattern for both third and fourth generation of cephalosporin with a value of approximately $77 \%$. Enterobacter cloacae also showed highly resistance pattern for both first and second generation of cephalosporin, with a value range between 57-86\%. As comparison, recent study by Kronenberg et al. ${ }^{18}$ also showed the increased resistance pattern of Gram-negative pathogen to the extended spectrum of cephalosporin (third and fourth generation).

The limitation of this study was the small range of population involved with short period of time to accomplish the study. It is recommended to perform a study with larger scale of population which involves many hospital around the district and more comprehensive method to get more accurate and reliable result as a current circumstances. The availability of medical documentation and antimicrobial susceptibility test results from laboratory was another limitation needed to be considered. The potential bias that can happen during this study was a selection bias of medical record. Since after the first procedure, the study should gather detailed information in the full hand-writing medical record (e.g unreadable); this could potentially make a bias. However, the crosscheck combination between medical record data with antimicrobial susceptibility data in the laboratory database, greatly reduced this bias. Detailed sorting, carefully date checking, and sequence of the test were the steps to eliminate the selection bias.

In conclusion, high resistance to cephalosporin occurs almost in all bacteria, specifically in first, third and fourth generation of cephalosporin among patients with chronic 
osteomyelitis, at Dr. Hasan Sadikin General Hospital.

Due to high resistance pattern of the bacteria to cephalosporin, it is useful to consider the antimicrobial susceptibility test result as the major point for antibiotic treatment in chronic osteomyelitis. Integrating and establishing patients' clinical demographic data and their antibiogram in concodance to chronic osteomyelitis is a mandatory. Furthermore, good communication between physian, laboratory staff, and pharmacist is also needed to generate a better patient's outcome and proper medical documentation.

\section{References}

1. Spellberg B, Lipsky BA. Systemic antibiotic therapy for chronic osteomyelitis in adults. Clin Infect Dis. 2012;54(3):393-407.

2. Stoesser N, Pocock J, Moore CE, Sona S, PutChhat H, Poda S, et al. The Epidemiology of Pediatric Bone and Joint Infections in Cambodia, 2007-11. J Trop Pediatr. 2013;59(1):36-42.

3. Hake M, Oh J, Kim J, Ziran B, Smith W, Hak D, et al. Difficulties and challenges to diagnose and treat post-traumatic long bone osteomyelitis. Eur J Orthop Surg Traumatol. 2015;25(1):1-3.

4. Kim P, Rosenblum B. Diagnosing Osteomyelitis. Podiatry Management. 2014;33(6):99-102.

5. Kasper DL, Longo DL. Osteomyelitis. In: Kasper DL, Longo DL, Braunwald E, Fauci AS, Hauser HL, Jameson JL, editors. Harrison's Principle of Internal Medicine. $16^{\text {th }}$ ed.New York: McGraw-Hill Companies, Inc; 2005. p. 745-9.

6. Hatzenbuehler J, Pulling TJ. Diagnosis and Management of Osteomyelitis. American Family Physician. 2011;84(9):1027-1033.

7. Johnson AP. Surveillance of antibiotic resistance. Phil Trans R Soc B. 2015;370(1670):20140080.

8. Cosgrove SE. The relationship between antimicrobial resistance and patient outcomes: mortality, length of hospital stay, and health care costs. Clin Infect Dis. 2006;42(2):82-9.

9. de Kraker MEA, Wolkewitz M, Davey PG,
Koller W, Berger J, Nagler J, et al. Clinical impact of antimicrobial resistance in European hospitals: excess mortality and length of hospital stay related to methicillin-resistant staphylococcus aureus bloodstream infections. Antimicrob Agents Chemother. 2011;55(4):1598-605.

10. World Health Organization. Antimicrobial resistance: global report on surveillance 2014. Geneva: WHO; 2014.

11. Brady RA, Leid JG, Calhoun JH, Costerton JW, Shirtliff ME. Osteomyelitis and the role of biofilms in chronic infection. FEMS Immunol Med Microbiol. 2008;52(1):1322.

12. Livorsi DJ, Daver NG, Atmar RL, Shelburne SA, White AC Jr, Musher DM. Outcomes of treatment for hematogenous Staphylococcus aureus vertebral osteomyelitis in the MRSA ERA. J Infect. 2008;57(2):128-31.

13. Gogia JS, Meehan JP, Di Cesare PE, Jamali AA. Local Antibiotic Therapy in Osteomyelitis. Semin Plast Surg. 2009;23(2):100-7.

14. Jorge LS, Chueire AG, Rossit ARB. Osteomyelitis: a current challenge. Braz J Infect Dis. 2010;14:(3):10-5.

15. Tuchscherr L, Kreis CA, Hoerr V, Flint L, Hachmeister M, Geraci J, et al. Staphylococcus aureus develops increased resistance to antibiotics by forming dynamic small colony variants during chronic osteomyelitis. J Antimicrob Chemother. 2016;71(2):438-48.

16. Gomes D, Pereira M, Bettencourt AF. Osteomyelitis: an overview of antimicrobial therapy. Braz J Pharm Sci. 2013;49(1):1327.

17. Holvoet K, Sampers I, Callens B, Dewulf J, Uyttendaele M. Moderate prevalence of antimicrobial resistance in Escherichia coli isolates from lettuce, irrigation water, and soil. Appl Environ Microbiol. 2013;79(21):6677-83.

18. Kronenberg A, Hilty M, Endimiani A, Muhlemann K. Temporal trends of extended-spectrum cephalosporinresistant Escherichia coli and Klebsiella pneumoniae isolates in in- and outpatients in Switzerland, 2004 to 2011. Euro surveill. 2013;18(21).pii:20484 